\title{
HPV vaccination has not increased sexual activity or accelerated sexual debut in a college-aged cohort of men and women
}

Andrew F. Brouwer ${ }^{1 *}$ (D), Rachel L. Delinger ${ }^{1 \dagger}$, Marisa C. Eisenberg ${ }^{1}$, Lora P. Campredon ${ }^{1}$, Heather M. Walline ${ }^{2}$, Thomas E. Carey ${ }^{2}$ and Rafael Meza ${ }^{1}$

\begin{abstract}
Background: The human papillomavirus (HPV) is the most common sexually transmitted infection and is linked to several types of cancer. HPV vaccination uptake in the U.S. is relatively low, despite the vaccine's high efficacy. Some parents of adolescents have concerns that vaccination will encourage sexual behavior and therefore choose not to vaccinate. Previous studies investigating vaccination and sexual behavior have included only young women and girls.

Methods: The objective of this study is to assess associations between HPV-vaccination and sexual behavior in a college-age cohort of both men and women. We analyzed questionnaire data collected from the Michigan HPV and Oropharyngeal Cancer Study, a cohort study designed to investigate HPV infection and its association with sexual behavior (data collected 2015-17, Ann Arbor, MI). Here, we consider vaccination status, sexual behavior, and substance use among 241 college-aged men and women. Logistic, Poisson, and Cox regression were used to determine the relationship between probability of sexual debut, number of sexual partners, and HPV vaccination status at baseline as well as between age at sexual debut and vaccination status at debut.

Results: HPV vaccination status was not significantly associated with an increased likelihood of sexual debut (odds ratio: 0.80 (95\% Cl: 0.41-1.58), decreased age of sexual debut (hazard ratio: 0.81 (95\% Cl: 0.65-1.00), nor an increased number of sexual partners (per year sexually active; incidence rate ratio: 1.27 (95\% Cl: $0.86-1.87)$ ) in this cohort, after controlling for age, race, sex, and substance use. Instead, race or alcohol use were independent predictors of sexual behavior.
\end{abstract}

Conclusions: Concerns about the influence of the HPV vaccine on sexual behavior are likely unfounded for both men and women. These results can aid in increasing vaccine acceptability, inform and strengthen physician recommendations, and ultimately reduce the burden of HPV and HPV-related cancers in the U.S.

Keywords: Human papillomavirus, Sexual behavior, HPV vaccine, College-aged population, Cohort study, M-HOC study

\footnotetext{
*Correspondence: brouweaf@umich.edu

${ }^{\dagger}$ Andrew F. Brouwer and Rachel L. Delinger contributed equally to this work.

${ }^{1}$ Department of Epidemiology, University of Michigan, 1415 Washington

Heights, Ann Arbor, Ml 48109, USA

Full list of author information is available at the end of the article
}

(c) The Author(s). 2019 Open Access This article is distributed under the terms of the Creative Commons Attribution 4.0 International License (http://creativecommons.org/licenses/by/4.0/), which permits unrestricted use, distribution, and reproduction in any medium, provided you give appropriate credit to the original author(s) and the source, provide a link to the Creative Commons license, and indicate if changes were made. The Creative Commons Public Domain Dedication waiver (http://creativecommons.org/publicdomain/zero/1.0/) applies to the data made available in this article, unless otherwise stated. 


\section{Background}

The human papillomavirus (HPV) is the most common sexually transmitted infection, with approximately 14 million new infections, both genital and oral, occurring every year in the United States; the vast majority of sexually active men and women will harbor an HPV infection at some point in their lifetime [1]. HPV infection with certain viral strains is linked to virtually every case of cervical cancer, $90 \%$ of anal cancers, and $40 \%$ of other genital cancers [2]. Additionally, while the burden of high-risk oral HPV infection in the population is low (around 4\%) [3], oropharyngeal squamous cell carcinomas have surpassed cervical cancers as the most common HPV-attributed cancers [2]-indeed, over $80 \%$ of oropharyngeal cancers at the University of Michigan Health System have been found to contain high-risk (oncogenic) HPV (defined in that study as genotypes $16,18,31,33,35,39,45,51,52$, 56, 58, 59, 66, 68, and 73) [4].

There are currently three prophylactic HPV vaccines with FDA approval: Gardasil (HPV types 6, 11, 16, and 18; approved in 2006 for young women, 2009 for young men), Cervarix (types 16 and 18; approved in 2009 for young women), and Gardasil 9 (types 6, 11, 16, 18, 31, 33, 45, 52, 58 ; approved in 2014 for young women and men). These vaccines target viral genotypes that are associated with a high risk of cervical cancer (HPV 16, 18, 31, 33, 45, 52, and 58) and those associated with genital warts (HPV 6 and 11). In the U.S., routine vaccination is recommended for 11-12 year old adolescents of both sexes. A two-dose vaccine schedule, given 6-12 months apart, is recommended for individuals who initiate the series between 9 and 14 years of age. A three-dose vaccination series is recommended for individuals who initiate the series between 15 and 26 years of age, as well as for immunocompromised persons [5]. Insurance in the US typically covers HPV vaccination for people within the recommended age ranges. Prevalence of vaccine genotypes is much lower in vaccinated people $[6,7]$.

Despite the high efficacy and safety of the vaccines [8], there has been low coverage and vaccine initiation rates among adolescents in the U.S., although coverage has recently been increasing, particularly in young men. In 2017 , only $68.6 \%$ of young women and $62.6 \%$ of young men had initiated the vaccine sequence in the U.S., while only $53.1 \%$ of young women and $44.3 \%$ of young men had received the full three recommended doses [9]. This coverage lags behind other adolescent vaccines [9] and falls considerably short of the goal set forth by the U.S. Department of Health and Human Services that $80 \%$ of adolescents be vaccinated against HPV. The CDC estimates that achieving this target uptake would prevent an additional 53,000 cases of cervical cancer [10].

Several factors have contributed to the low HPV vaccine uptake. General barriers to adolescent vaccine uptake include fewer visits to healthcare providers, incorrect beliefs about vaccines and vaccine safety, parental attitudes, and missed opportunities for vaccine administration [11-13]. Providers often fail to strongly endorse the HPV vaccination-particularly for patients who were younger, male, or from an ethnic minority $[14,15]$. Various personal and political stances were also found to be barriers for strong provider recommendations. Additionally, because HPV is a sexually-transmitted infection, many parents have expressed concern that HPV vaccination may encourage children to become sexually active or promote promiscuous or riskier sexual behavior. Several studies assessing parental opinions of the HPV vaccine have found that up to $20 \%$ of parents believe the vaccination would lead to riskier sexual behavior in the future [16]. Parents who cite attitudinal barriers to vaccination are less accepting of the vaccine than parents who cite logistical barriers to vaccination their children [17]. These factors are a major obstacle in HPV vaccine uptake. Increasing acceptability and understanding among parents, as well as strengthening provider recommendations, will be necessary for expanding vaccine coverage and reducing disease burden [18].

Previous studies have found no evidence that HPV vaccination impacts sexual behavior, risk perception, or sexually transmitted infection incidence [19-26]. However, these studies have focused only on adolescent and young adult women, largely because cultural concerns about vaccination affecting sexual behavior have been muted for men [27], which is reflective of the sexual double-standard. Here, we measure the association between HPV vaccination and sexual behaviors in male and female college-aged students at the University of Michigan and college-aged participants living in Ann Arbor, Michigan and the surrounding communities. The study participants are part of the Michigan HPV and Oropharyngeal Cancer (M-HOC) study, which aims to understand the natural history of oral HPV infection by collecting longitudinal questionnaire and biological data on a cohort from the Ann Arbor, Michigan area.

\section{Methods}

\section{Ethics, consent and permissions}

Written and documented informed consent was obtained from all participants. Participant ID numbers were assigned to ensure participant confidentiality. The University of Michigan IRB approved consent documents and study protocol (HUM00090236).

\section{Study participants}

Electronic questionnaires were administered in a private room to a convenience sample of college students and adult residents of Ann Arbor, Michigan and the immediate surrounding areas. Study participants were recruited at University of Michigan campus dormitories, through fliers, and through the UM Health Research website. 
Volunteers over the age of 18 who were willing to return every 3-4 months for 3 years for follow-up visits were invited to enroll. Participants with a history of head and neck cancer were not eligible. Additional information is available in our study protocol [28]. Enrollment has been completed. Data analysis here was restricted to the baseline questionnaire of college-aged (18-22) participants $(N=241)$ to investigate the impact of adolescent HPV vaccination on sexual behavior (data collected 2015-17, analyzed 2017-19). Recruitment in this age range targeted younger people to maximize retention over the planned 3year study. Certain analyses are restricted to participants who had or did not have the HPV vaccine $(N=217$; those who were unsure or did not answer were excluded).

\section{Questionnaire}

A baseline questionnaire was administered to each participant at their initial visit. The questionnaire was designed to individually assess a variety of topics including demographics, vaccination, sexual health and behavior, and alcohol and drug use. Sexual behavior questions separately assessed current and past experiences of vaginal, oral, and anal sex.

\section{Exposure and outcomes}

Three key outcomes were considered: occurrence of sexual debut (the first experience of vaginal, oral, or anal sex), number of sexual partners (lifetime number or per year sexually active), and age at sexual debut. Sexual behavior variables define sexual contact as vaginal intercourse, oral sex, or anal sex and define a sexual partner as an individual with whom one engages in one or more of these acts.

The main exposure variables in this analysis were vaccination status at study baseline ("Have you been vaccinated against human papillomavirus (HPV)?") and at sexual debut as determined by reported age at vaccination ("What age did you get your first dose [of HPV vaccine]?") and at sexual debut ("How old were you when you first had [vaginal/oral/anal] sex?”). Participants were considered vaccinated at sexual debut if their reported age at first dose of an HPV vaccine was less than or equal to their age of sexual debut. Anecdotally, participants contacting parents for vaccination status confirmation during the baseline interview was not unusual.

Vaccination at baseline was used as the exposure for the occurrence of sexual debut and number of sexual partner outcomes because we were interested in whether there was an association between them, irrespective of the timing of vaccination and sexual debut. However, when analyzing the impact of vaccination on the age of sexual debut, we recognize that participants may have been vaccinated after sexual debut but before their baseline visit. Hence, we use vaccination at the time of sexual debut as the exposure variable for this analysis.

\section{Statistical analysis}

Descriptive analysis was performed to assess participant characteristics and behaviors from questionnaire data, including demographics, vaccination, sexual behavior, and substance-use. Differences in numbers and ages by vaccination status or gender were assessed by t-test or ANOVA, while differences in distribution of categorical or binary variables were assessed by chi-square test. $P$ values are omitted if test assumptions appear to be violated. Logistic regression was used to assess the relationship between baseline HPV vaccination status and sexual debut; results are reported as odds ratios (i.e., exponentiated model coefficients). Poisson regression, with an offset of (the log of) time since sexual debut, was used to determine the association between baseline HPV vaccination status and number of sexual partners (per year since sexual debut); results are presented as incidence ratios (i.e., exponentiated model coefficients). When age at sexual debut was the same as the current age, we assumed that the time since sexual debut was $1 / 3$ years (consistent with uniform distribution of events). Cox proportional hazard models and the log-rank test were used to assess the relationship between HPV vaccination status at sexual debut and age at sexual debut. Results are presented as hazard ratios (i.e., exponentiated model coefficients). Models were adjusted for various demographic and behavioral characteristics, including age, sex, race, alcohol, and drug use at the time of the survey. Both multivariate analyses were done on the subset of participants who reported having or not having the HPV vaccination at baseline. All analyses were done in $\mathrm{R}$ v3.4. Statistical significance is reported at level $\alpha=0.05$; associations in the multivariate models with $p$-values less than $\alpha=0.1$ are noted as approaching significance to avoid overlooking associations that were not significant because of limited sample size.

\section{Results \\ Population characteristics and differences by vaccination status and gender}

At completion of study enrollment, 241 college-aged participants were enrolled into the study and completed the baseline questionnaire. Participant demographics, vaccination status, substance use, and sexual behaviors are shown in Table 1. The mean age of the college-aged cohort was 18.9 years. The majority of participants were female $(70 \%)$ and white (54\%). A majority of participants (63\%) had previous sexual experience at baseline, of whom $77 \%$ had a history of vaginal sex, $96 \%$ had a history of oral sex, and $20 \%$ had a history of anal sex. The mean age of sexual debut was 16.6 years; however, this value is likely an underestimate of the (eventual average) age of sexual debut for this cohort because some participants may debut after the survey. 
Table 1 Characteristics of the M-HOC college-age cohort and stratification by HPV vaccination status and gender

\begin{tabular}{|c|c|c|c|c|c|c|c|c|c|c|c|c|}
\hline \multirow[b]{2}{*}{ Variable } & \multicolumn{2}{|c|}{ Full Cohort } & \multicolumn{2}{|c|}{ Vaccinated } & \multicolumn{2}{|c|}{ Unvaccinated } & \multirow[b]{2}{*}{$p$-value ${ }^{a}$} & \multicolumn{2}{|c|}{ Female } & \multicolumn{2}{|c|}{ Male } & \multirow[b]{2}{*}{$p$-value ${ }^{a}$} \\
\hline & $\bar{N}$ & Value & $\bar{N}$ & Value & $\bar{N}$ & Value & & $\bar{N}$ & Value & $\bar{N}$ & Value & \\
\hline Age & 241 & $18.9(1.0)$ & 158 & $18.8(1.0)$ & 59 & $19.1(1.0)$ & 0.09 & 168 & $18.8(1.0)$ & 73 & $19.0(1.1)$ & 0.17 \\
\hline Female & 241 & $70 \%(168)$ & 158 & $74 \%(117)$ & 59 & $66 \%(39)$ & 0.25 & 168 & $100 \%(168)$ & 73 & $0 \%(0)$ & - \\
\hline Race & 241 & & 158 & & 59 & & - & 168 & & 73 & & - \\
\hline White & & $54 \%(131)$ & & $56 \%(88)$ & & $61 \%(36)$ & & & $56 \%(94)$ & & $52 \%(37)$ & \\
\hline Asian & & $31 \%(74)$ & & $30 \%(47)$ & & $24 \%(14)$ & & & $29 \%(49)$ & & $35 \%(25)$ & \\
\hline Black & & $6 \%(14)$ & & $4 \%(7)$ & & $8 \%(5)$ & & & $5 \%(9)$ & & $7 \%(5)$ & \\
\hline Other/multiracial & & $8 \%(20)$ & & $9 \%(14)$ & & $7 \%(4)$ & & & $10 \%(16)$ & & $6 \%(4)$ & \\
\hline Vaccinated & 217 & $73 \%(158)$ & 158 & $100 \%(153)$ & 59 & $0 \%(0)$ & - & 156 & $75 \%(117)$ & 61 & $67 \%(41)$ & 0.25 \\
\hline Age at first vaccine dose & - & - & 102 & $15.5(2.4)$ & - & - & - & 79 & $15.0(2.5)$ & 22 & $16.7(1.6)$ & $<0.001$ \\
\hline Current smoker & 231 & $1 \%(2)$ & 158 & $1 \%(1)$ & 59 & $2 \%(1)$ & - & 168 & $0 \%(0)$ & 73 & $3 \%(2)$ & - \\
\hline Alcohol: current user & 231 & $65 \%(157)$ & 158 & $68 \%(104)$ & 59 & $63 \%(35)$ & 0.49 & 168 & $65 \%(109)$ & 73 & $66 \%(48)$ & 0.90 \\
\hline Alcohol: ever binge drinker ${ }^{\mathrm{b}}$ & 231 & $15 \%(37)$ & 158 & $16 \%(25)$ & 59 & $15 \%(9)$ & 0.92 & 168 & $11 \%(19)$ & 73 & $25 \%(18)$ & 0.01 \\
\hline Marijuana: ever user & 231 & $38 \%(91)$ & 158 & $37 \%(58)$ & 59 & $41 \%(24)$ & 0.59 & 168 & $36 \%(60)$ & 73 & $42 \%(31)$ & 0.32 \\
\hline Has had any sex & 231 & $63 \%(151)$ & 158 & $64 \%(101)$ & 59 & $61 \%(36)$ & 0.69 & 168 & $62 \%(104)$ & 73 & $64 \%(47)$ & 0.71 \\
\hline \multicolumn{13}{|l|}{ If has had sex, has had } \\
\hline Vaginal & 151 & $77 \%(116)$ & 101 & $77 \%(78)$ & 36 & $75 \%(27)$ & 0.79 & 104 & $78 \%(81)$ & 47 & $74 \%(35)$ & 0.70 \\
\hline Oral & 151 & $96 \%(145)$ & 101 & $97 \%(93)$ & 36 & $94 \%(31)$ & - & 104 & $97 \%(101)$ & 47 & $94 \%(44)$ & - \\
\hline Anal & 151 & $20 \%(30)$ & 101 & $21 \%(21)$ & 36 & $22 \%(8)$ & 0.86 & 104 & $17 \%(18)$ & 47 & $26 \%(12)$ & 0.24 \\
\hline \multicolumn{13}{|l|}{ Lifetime number of sexual partners } \\
\hline Any & 241 & $2.2(3.7)$ & 158 & $2.7(4.2)$ & 59 & $1.9(2.7)$ & 0.15 & 168 & $2.1(3.2)$ & 73 & $2.8(4.6)$ & 0.24 \\
\hline Vaginal sex & 241 & $1.6(3.1)$ & 158 & $1.9(3.5)$ & 59 & $1.2(2.3)$ & 0.09 & 168 & $1.7(2.9)$ & 73 & $1.4(3.6)$ & 0.58 \\
\hline Oral sex & 241 & $2.3(3.6)$ & 158 & $2.7(4.1)$ & 59 & $1.8(2.7)$ & 0.09 & 168 & $2.1(3.0)$ & 73 & $2.7(4.7)$ & 0.34 \\
\hline Anal sex & 241 & $0.2(0.8)$ & 158 & $0.3(0.8)$ & 59 & $0.2(0.6)$ & 0.64 & 168 & $0.1(0.5)$ & 73 & $0.4(1.2)$ & 0.08 \\
\hline Age at sexual debut & 150 & $16.6(1.7)$ & 101 & $16.5(1.7)$ & 36 & $16.9(1.8)$ & 0.18 & 104 & $16.7(1.6)$ & 46 & $16.5(1.9)$ & 0.58 \\
\hline Uses protection during vaginal sex & 114 & & 78 & & 26 & & - & 81 & & 33 & & - \\
\hline Always & & $47 \%(54)$ & & $41 \%(32)$ & & $62 \%(16)$ & & & $46 \%(37)$ & & $52 \%(17)$ & \\
\hline Most of the time & & $32 \%(37)$ & & $37 \%(29)$ & & $23 \%(6)$ & & & $33 \%(27)$ & & $30 \%(10)$ & \\
\hline Sometimes & & $9 \%(10)$ & & $8 \%(6)$ & & $8 \%(2)$ & & & $11 \%(9)$ & & $2 \%(1)$ & \\
\hline Rarely & & $6 \%(7)$ & & $9 \%(7)$ & & $0 \%(0)$ & & & $5 \%(4)$ & & $9 \%(3)$ & \\
\hline Never & & $5 \%(6)$ & & $5 \%(4)$ & & $8 \%(2)$ & & & $5 \%(4)$ & & $6 \%(2)$ & \\
\hline Uses protection during oral sex & 139 & & 96 & & 31 & & - & 96 & & 43 & & - \\
\hline Always & & $1 \%(1)$ & & $1 \%(1)$ & & $0 \%(0)$ & & & $1 \%(1)$ & & $0 \%(0)$ & \\
\hline Most of the time & & $0 \%(0)$ & & $0 \%(0)$ & & $0 \%(0)$ & & & $0 \%(0)$ & & $0 \%(0)$ & \\
\hline Sometimes & & $4 \%(5)$ & & $4 \%(4)$ & & $3 \%(1)$ & & & $4 \%(4)$ & & $2 \%(1)$ & \\
\hline Rarely & & $8 \%(11)$ & & $9 \%(10)$ & & $0 \%(0)$ & & & $10 \%(10)$ & & $2 \%(1)$ & \\
\hline Never & & $88 \%(122)$ & & $84 \%(81)$ & & $97 \%(30)$ & & & $84 \%(81)$ & & $95 \%(41)$ & \\
\hline Uses protection during anal sex & 30 & & 21 & & 8 & & - & 18 & & 12 & & - \\
\hline Always & & $33 \%(10)$ & & $29 \%(6)$ & & $50 \%(4)$ & & & $28 \%(5)$ & & $42 \%(5)$ & \\
\hline Most of the time & & $17 \%(5)$ & & $14 \%(3)$ & & $13 \%(1)$ & & & $17 \%(3)$ & & $17 \%(2)$ & \\
\hline Sometimes & & $7 \%(2)$ & & $10 \%(2)$ & & $0 \%(0)$ & & & $11 \%(2)$ & & $0 \%(0)$ & \\
\hline Rarely & & $3 \%(1)$ & & $5 \%(1)$ & & $0 \%(0)$ & & & $0 \%(0)$ & & $8 \%(1)$ & \\
\hline Never & & $40 \%(12)$ & & $43 \%(9)$ & & $38 \%(3)$ & & & $44 \%(8)$ & & $33 \%(4)$ & \\
\hline
\end{tabular}

Values are \% (number) out of $\mathrm{N}$ or age/number (sd). Only 217 of the 241 participants indicated vaccination status. Because participants could choose to not answer questions, sums of categorical entries may not equal the corresponding N. The M-HOC (Michigan HPV and Oropharyngeal Cancer) study was conducted in 2015-17 in Ann Arbor, MI

a: $p$-value for difference in means between vaccinated and unvaccinated populations, given by t-test (continuous or integer) or chi-square (binary and categorical). Boldface indicate $p<0.05$

b: Habitual consumption of at least 6-9 drinks in a sitting 
Most participants (90\%) indicated their HPV vaccination status. Comparisons of participant characteristics and behaviors by vaccination status and gender are also shown in Table 1. Vaccinated individuals were more likely to be female, although this difference was not statistically significant. No significant differences in demographic characteristics were found between vaccination groups, nor in terms of substance use or use of protection during sex. The unadjusted difference in the mean number of vaginal sexual partners achieved statistical significance, with vaccinated individuals reporting a higher mean number of sexual partners, though it would be misleading to interpret this statistic without adjusting for covariates and time since sexual debut (see below). Men and women differed slightly (but not statistically significantly) in proportion vaccinated (67\% vs. $75 \%)$, and men were vaccinated later on average (age 16.7 vs. 15.0). There were no significant differences in sexual behavior by gender (Table 1 ).

\section{Multivariate models}

We analyzed sexual debut, the number of sexual partners, and age at sexual debut, controlling for age, sex, race, substance use, and vaccination status (Table 2). Analyses likelihood of sexual debut and number of sexual partners were restricted to participants who selfreported their HPV status $(N=217)$; analysis of age at sexual debut was restricted to participants who additionally reported age at vaccination and age at sexual debut $(N=161)$. Increased likelihood of sexual debut was associated with age and reporting current alcohol use; being white approached significance. (Note: although the positive association between being white and reporting current alcohol use approached significance $(p=0.07)$, the regression accounts for the correlation structure.
Hence, these covariates are independent predictors of sexual debut). Vaccination status was not significantly associated with probability of sexual debut. Next, by using a Poisson regression with an offset of time since sexual debut, we were able to adjust our analysis of number of sexual partners to account for the most important factor, namely, the length of time participants had been sexually active. After this adjustment, the number of sexual partners was strongly positively associated with current alcohol use and weakly (in magnitude), negatively associated with being white. Vaccination status also approached statistical significance in this analysis; however, unlike in Table 1, it was weakly associated with fewer sexual partners after adjusting for covariates.

For age of sexual debut, values are multiplicative effects on the underlying hazard rate, meaning that larger hazard ratios are associated with earlier age of sexual debut (Table 2). This analysis was restricted to the subset of participants who reported their age at vaccination or not being vaccinated; among this subset, 83 participants reported vaccination before or the same age as debut, and 78 participants reported vaccination after debut or no vaccination. There were 110 sexual debuts in this subset. Being white was associated with an earlier sexual debut, and reporting current alcohol use at baseline neared significance. But, vaccination status at sexual debut was not significant. A Kaplan-Meier plot of sexual debut stratified by HPV-vaccination status at sexual debut (Fig. 1) illustrates the finding that HPV-vaccination status at debut is not significantly associated with age of sexual debut (log-rank test, $p=0.30$ ). Analogous multivariate models of probability of sexual debut, number of sexual partners and age of sexual debut for vaginal, oral, and anal sex are included in the Additional file 1. These models have largely similar interpretations, although the

Table 2 Multivariate analyses predicting number of sexual partners and age at sexual debut

\begin{tabular}{|c|c|c|c|c|c|c|c|c|c|}
\hline \multirow[b]{2}{*}{ Covariate } & \multicolumn{3}{|c|}{ Probability of sexual debut } & \multicolumn{3}{|c|}{ Number of sexual partners (per year) } & \multicolumn{3}{|c|}{ Age at sexual debut } \\
\hline & Odds ratio & $95 \% \mathrm{Cl}$ & $p$-value & Incidence ratio & $95 \% \mathrm{Cl}$ & $p$-value & Hazard ratio & $95 \% \mathrm{Cl}$ & $p$-value \\
\hline Intercept & 0.61 & $(0.19,1.96)$ & 0.41 & 1.47 & $(0.98,2.19)$ & 0.06 & - & - & - \\
\hline Age (per year over 18) & 1.48 & $(1.05,2.14)$ & 0.03 & 0.97 & $(0.89,1.05)$ & 0.42 & 0.88 & $(0.73,1.07)$ & 0.21 \\
\hline Female & 0.85 & $(0.42,1.68)$ & 0.65 & 0.99 & $(0.82,1.20)$ & 0.94 & 0.90 & $(0.58,1.42)$ & 0.65 \\
\hline Race: white & 1.78 & $(0.98,3.27)$ & 0.06 & 0.75 & $(0.63,0.89)$ & 0.001 & 1.51 & $(1.01,2.28)$ & 0.04 \\
\hline Alcohol: current drinker & 2.73 & $(1.36,5.56)$ & 0.001 & 1.81 & $(1.36,2.43)$ & $<0.001$ & 1.65 & $(0.98,2.80)$ & 0.06 \\
\hline Alcohol: binge drinker & 1.71 & $(0.64,5.12)$ & 0.30 & 0.99 & $(0.79,1.22)$ & 0.92 & 1.34 & $(0.82,2.22)$ & 0.25 \\
\hline Marijuana: ever user & 1.27 & $(0.60,2.70)$ & 0.53 & 1.06 & $(0.86,1.32)$ & 0.57 & 1.13 & $(0.73,1.75)$ & 0.58 \\
\hline \multicolumn{10}{|l|}{ Vaccinated } \\
\hline At baseline & 0.80 & $(0.41,1.58)$ & 0.52 & 0.81 & $(0.65,1.00)$ & 0.06 & - & - & - \\
\hline At sexual debut & - & - & - & - & - & - & 1.27 & $(0.86,1.87)$ & 0.23 \\
\hline
\end{tabular}

The probability of sexual debut is analyzed by logistic regression; values are given as odds ratios (exponentiated model coefficients). The number of sexual partners is analyzed with Poisson regression with an offset of (the log of) number of years sexually active; values are incidence rate ratios (exponentiated model coefficients). The age at sexual debut is analyzed by a Cox proportional hazard model; results given as hazard ratios (exponentiated model coefficients). Boldface indicate $p<0.05$. Study conducted in 2015-17, Ann Arbor, MI 


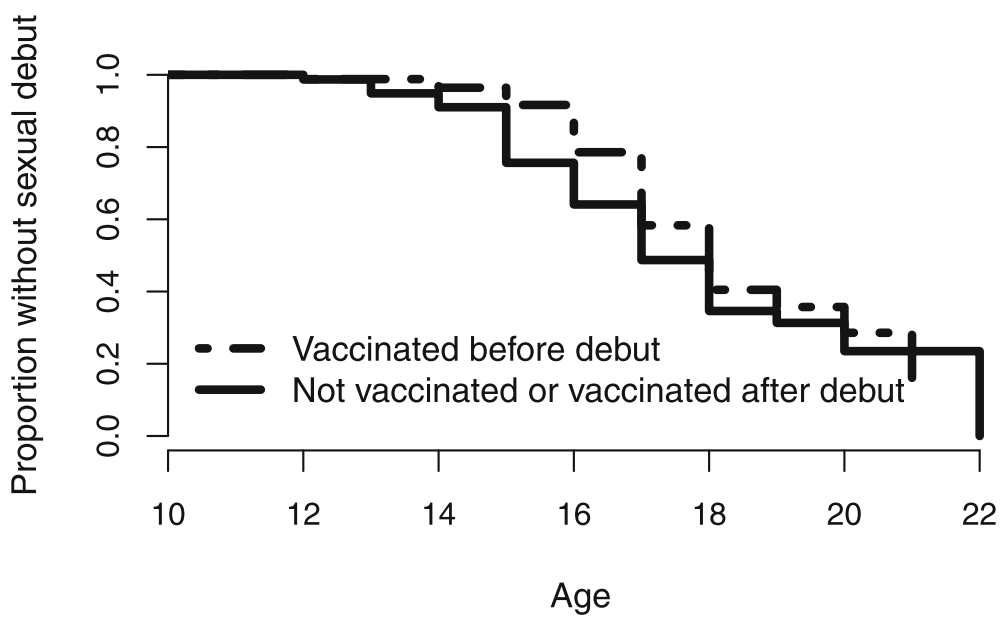

Fig. 1 Kaplan-Meier plot of age at sexual debut stratified by HPV vaccination status at debut. No statistically significant difference between the curves was detected (log-rank test, $p=0.30$ )

probability and age of vaginal sexual debut were more associated with current marijuana use than with current alcohol use. Binge drinking was not associated with any of the outcomes after accounting for current alcohol use.

\section{Discussion}

Our analysis of behavioral survey data for a college-age cohort reveals a nuanced story of sexual behavior and HPV vaccination. Although vaccinated participants had more sexual partners overall, after adjusting for time since sexual debut and other covariates, we found instead that a weak association between HPV-vaccination and fewer sexual partners approached statistical significance. Although we expect that this association may be spurious, it may also be that vaccination uptake and fewer sexual partners are both associated with risk avoidance behavior. Regardless, we interpret this finding as evidence that HPV-vaccination is not associated with increased sexual activity. Vaccination status at sexual debut was not significantly associated with the probability of sexual debut or the age at sexual debut, though alcohol use or being white were. Gender was not significantly associated with any of the main outcomes but was significant in certain sexact-specific outcomes.

This study contributes to the emerging evidence that HPV vaccination does not promote sexual behavior in young adults. Previous studies have reported no differences in the number of sexual partners [19, 25], age of sexual debut [19], sexual activity and condom use [20], risk perceptions $[22,26]$, STI and pregnancy incidence or testing $[21,23,24]$ in girls and young women. Many parents have hesitated to vaccinate their children against HPV out of fear of risky or adverse sexual behavior outcomes later in life [16]. For example, a 2009 study by Kahn et al. [29] found that only $48 \%$ of mothers intended to vaccinate daughters under the age of 13 , the target age group for HPV vaccination. Therefore, evidence refuting these concerns has clinical implications, since parental concern has found to be an important barrier to physician recommendation of vaccination [30]. Physician biases and concerns related to teen sexual behavior have also been reported, particularly as a barrier to male vaccination [17, 31]. Since physician recommendations, as well as parental acceptance of the vaccine, are vital to vaccine uptake [18], strengthening the evidence for the lack of association between HPV vaccination and subsequent sexual behavior is imperative.

Our finding that sexual behavior (number of partners and sexual debut) is strongly associated with alcohol use is consistent with many previous studies citing a link between drinking and increased sexual activity. For example, the Harvard College Alcohol Study (CAS) provided national data showing that college students who engaged in binge drinking were 2-3 times more likely to engage in unplanned sex and unprotected sex [32]. In this study, we found that binge drinking was not associated with the outcomes after adjusting for any alcohol consumption, although the definition of binge drinking varied slightly between studies. Moreover, Metrik et al. [33] found both alcohol use and marijuana use were independently associated with a greater likelihood of sexual encounters; we found that alcohol, though not marijuana, use was associated with our sexual behavior outcomes in our cohort.

This study provided a first look at the cohort data generated by the M-HOC study, which, at its completion, has the potential to inform many other aspects of HPV infection, including the relationship between longitudinal sexual behaviors, including debut, and oral HPV infection incidence, prevalence, and clearance. This study is the first to investigate the impact of HPV vaccination on sexual behavior that we are aware of that has included men. 
Further, targeting a college-aged sample can investigate any effects that vaccination may have closer to the age of sexual debut. Studying both short-term and long-term effects of vaccination status is critical to understanding the interplay between sexual behavior and vaccination and ultimately increasing vaccine uptake.

Our analysis is based on self-reported questionnaire data, and it is not possible to independently confirm the reported sexual and substance-use behaviors or vaccination status. Since the majority of vaccinated participants in our cohort were vaccinated years prior to the baseline questionnaire, it is possible that individuals misremembered or were unsure of their HPV vaccination status. Although we accounted for HPV status at sexual debut by including it as an exposure, sexual debut and vaccination can both be viewed as survival processes, and thus future work could take a competing risks approach to assessing associations between them. Moreover, in this retrospective analysis, we tested for associations between substance use at study baseline and prior sexual debut; consequently, these associations are inherently not causative. Too, the location and recruiting method of this study may lead to a generalizability limitation. This cohort is composed of college-aged individuals, the majority of whom are white and attend a large university. These characteristics could potentially influence behavioral decisions in a manner not generalizable to other individuals of the same age. Moreover, because of the timing of vaccine introduction, this cohort was vaccinated later (mean age 15.5) than younger cohorts will likely be, since they did not (and largely could not) have received the vaccine as now recommended. Nonetheless, our results provide additional evidence of the lack of association between HPV vaccination and sexual behaviors across different populations.

\section{Conclusion}

Our findings contribute to a fast-growing body of literature that demonstrates that HPV vaccination does not impact sexual behavior of adolescents or college-aged individuals. These studies are an important step to increasing vaccine uptake in the United States. By educating parents of adolescents as well as increasing awareness among physicians, physician recommendations can more accurately reflect the true benefits and risks of HPV vaccination, potentially leading to increased vaccine acceptability. We will continue follow-up of this cohort to further investigate behavioral outcomes and the natural history of HPV.

\section{Additional file}

Additional file 1: Table S1. Characteristics of the M-HOC college-age cohort stratified by the number of sexual partners. Table S2.

Characteristics of the M-HOC college-age cohort stratified by sexual experience. Table S3. Multivariate analyses predicting age at vaginal sexual debut and lifetime number of vaginal sexual partners. Table S4. Multivariate analyses predicting age at oral sexual debut and lifetime number of oral sexual partners. Table S5. Multivariate analyses predicting age at anal sexual debut and lifetime number of anal sexual partners. (DOCX $49 \mathrm{~kb})$

Abbreviations

ANOVA: analysis of variance; CDC: Centers for Disease Control and Prevention; FDA: Food and Drug Administration; HPV: human papillomavirus; M-HOC: Michigan HPV and Oropharyngeal Cancer); UM UM: University of Michigan

\section{Acknowledgments}

We would like to thank the Michigan HPV and Oropharyngeal Cancer (MHOC) study team for making this work possible.

\section{Authors' contributions}

Conceptualization: MCE, TEC, RM. Analysis: AFB, RLD. Interpretation: AFB, RLD, MCE, LPC, HMW, TEC, RM. Draft preparation: AFB, RLD. Review and editing: AFB, RLD, MCE, LPC, HMW, TEC, RM. All authors have read and approved the manuscript.

\section{Funding}

This work was supported by National Institutes for Health grant U01CA182915. The study sponsor had no role in study design; collection, analysis, and interpretation of data; writing the report; nor the decision to submit the report for publication.

\section{Availability of data and materials}

The datasets generated and/or analyzed during the current study are not publicly available because of participant privacy concerns but are available from the corresponding author on reasonable request (IRB approval or a data use agreement may be required).

\section{Ethics approval and consent to participate}

The University of Michigan IRB approved consent documents and study protocol (HUM00090236). Written and documented informed consent was obtained from all participants.

Consent for publication

Not applicable.

\section{Competing interests}

The authors declare that they have no competing interests.

\section{Author details}

${ }^{1}$ Department of Epidemiology, University of Michigan, 1415 Washington Heights, Ann Arbor, Ml 48109, USA. ²Department of Otolaryngology,

University of Michigan, 1500 E. Medical Center Dr., Ann Arbor, MI 48109, USA.

Received: 15 January 2019 Accepted: 9 June 2019

Published online: 25 June 2019

\section{References}

1. Centers for Disease Control and Prevention. Genital HPV Infection - Fact Sheet. 2017. URL: https://www.cdc.gov/std/hpv/stdfact-hpv.htm.

2. Jemal A, Simard EP, Dorell C, Noone AM, Markowitz LE, Kohler B, et al. Annual report to the nation on the status of Cancer, 1975-2009, featuring the burden and trends in human papillomavirus (HPV)-associated cancers and HPV vaccination coverage levels. J Natl Cancer Inst. 2013;105(3):175-201.

3. McQuillan G, Kruszon-Moran D, Markowitz LE, Unger ER, Paulose-Ram R. Prevalence of HPV in adults aged 18-69: United States, 2011-14. NCHS Data Brief. 2017;280:1-8

4. Walline HM, Komarck C, McHugh JB, Byrd SA, Spector ME, Hauff SJ, et al. High-risk human papillomavirus detection in oropharyngeal, nasopharyngeal, and Oral cavity cancers. J Am Med Assoc Otolaryngol Head Neck Surg. 2013;139(12):1320-7.

5. Meites E, Kempe A, Markowitz LE. Use of a 2-dose schedule for human papillomavirus vaccination updated recommendations of the advisory 
committee on immunization practices. MMWR Morb Mortal Wkly Rep. 2016; 65(49):1405-8.

6. Brouwer AF, Eisenberg MC, Carey TE, Meza R. Multisite HPV infections in the United States (NHANES 2003-2014): an overview and synthesis. Prev Med. 2019;123:288-98.

7. Markowitz LE, Liu G, Hariri S, Steinau M, Dunne EF, Unger ER. Prevalence of HPV after introduction of the vaccination program in the United States. Pediatrics. 2016;137(2):e20151968.

8. Kash N, Lee M, Kollipara R, Downing C, Guidry J, Tyring S. Safety and efficacy data on vaccines and immunization to human papillomavirus. J Clin Med. 2015;4(4):614-33.

9. Walker TY, Elam-Evans LD, Yankey D, Markowitz LE, Williams CL, Mbaeyi SA, et al. National, regional, state, and selected local area vaccination coverage among adolescents aged 13-17 years-United States, 2017. MMWR Morb Mortal Wkly Rep. 2018;67(33):909-17.

10. Orenstein WA, Gellin BG, Beigi RH, Despres S, Lynfield R, Maldonado Y, et al. Overcoming barriers to low HPV vaccine uptake in the United States: recommendations from the National Vaccine Advisory Committee: approved by the National Vaccine Advisory Committee on June 9, 2015. Public Health Rep. 2016;131(1):17-25.

11. Olshen E, Woods ER, Austin SB, Luskin M, Bauchner H. Parental acceptance of the human papillomavirus vaccine. J Adolesc Health. 2005;37(3):248-51.

12. Roberts JR, Thompson D, Rogacki B, Hale JJ, Jacobson RM, Opel DJ, et al. Vaccine hesitancy among parents of adolescents and its association with vaccine uptake. Vaccine. 2015;33(14):1748-55.

13. Kornides ML, McRee AL, Gilkey MB. Parents who decline HPV vaccination: who later accepts and why? Acad Pediatr. 2018;18(2):S21-2.

14. Gilkey MB, McRee AL. Provider communication about HPV vaccination: a systematic review. Hum Vaccin Immunother. 2016;12(6):1454-68.

15. Finney Rutten $L$, St. Sauver $J$, Beebe TJ, Wilson PM, Jacobson DJ, Fan C, et al. Association of both consistency and strength of self-reported clinician recommendation for HPV vaccination and HPV vaccine uptake among 11to 12-year-old children. Vaccine. 2017;35(45):6122-8.

16. Forster A, Wardle J, Stephenson J, Waller J. Passport to promiscuity or lifesaver: press coverage of HPV vaccination and risky sexual behavior. J Health Commun. 2010;15(2):205-17.

17. Donahue KL, Stupiansky NW, Alexander AB, Zimet GD. Acceptability of the human papillomavirus vaccine and reasons for non-vaccination among parents of adolescent sons. Vaccine. 2014;32(31):3883-5.

18. Vollrath $\mathrm{K}$, Thul $\mathrm{S}$, Holcombe J. Meaningful methods for increasing human papillomavirus vaccination rates: an integrative literature review. J Pediatr Health Care. 2018;32(2):119-32

19. Liddon NC, Leichliter JS, Markowitz LE. Human papillomavirus vaccine and sexual behavior among adolescent and young women. Am J Prev Med. 2012;42(1):44-52.

20. Forster AS, Marlow LA, Stephenson J, Wardle J, Waller J. Human papillomavirus vaccination and sexual behaviour: cross-sectional and longitudinal surveys conducted in England. Vaccine. 2012;30(33):4939-44.

21. Bednarczyk RA, Davis R, Ault K, Orenstein W, Omer SB. Sexual activity-related outcomes after human papillomavirus vaccination of 11- to 12-year-olds. Pediatrics. 2012;130(5):798-805.

22. Mayhew A, Mullins TLK, Ding L, Rosenthal SL, Zimet GD, Morrow C, et al. Risk perceptions and subsequent sexual behaviors after HPV vaccination in adolescents. Pediatrics. 2014;133(3):404-11.

23. Smith LMM, Kaufman JSP, Strumpf ECP, Lévesque LEP. Effect of human papillomavirus (HPV) vaccination on clinical indicators of sexual behaviour among adolescent girls: the Ontario grade 8 HPV vaccine cohort study. Can Med Assoc J. 2015;187(2):E74-81.

24. Jena $A B$, Goldman DP, Seabury SA. Incidence of sexually transmitted infections after human papillomavirus vaccination among adolescent females. J Am Med Assoc Intern Med. 2015;175(4):617-23.

25. Ogilvie GS, Phan F, Pederson HN, Dobson SR, Naus M, Saewyc EM. Population-level sexual behaviours in adolescent girls before and after introduction of the human papillomavirus vaccine (2003-2013). Can Med Assoc J. 2018;190(41):E1221-6.

26. Mullins TLK, Zimet GD, Rosenthal SL, Morrow C, Ding L, Huang B, et al. Human papillomavirus vaccine-related risk perceptions and subsequent sexual behaviors and sexually transmitted infections among vaccinated adolescent women. Vaccine. 2016;34(34):4040-5.
27. Mayer MK, Reiter PL, Zucker RA, Brewer NT. Parents' and sons' beliefs in sexual disinhibition after human papillomavirus vaccination. Sex Transm Dis. 2013;40(10):822-8

28. Eisenberg MC, Campredon LP, Brouwer AF, et al. Dynamics and determinants of HPV infection: the Michigan HPV and oropharyngeal Cancer (M-HOC) study. BMJ Open. 2018;8:e021618.

29. Kahn JA, Ding L, Huang B, Zimet GD, Rosenthal SL, Frazier AL. Mothers' intention for their daughters and themselves to receive the human papillomavirus vaccine: a National Study of nurses. Pediatrics. 2009; 123(6):1439-45.

30. Kahn JA, Zimet GD, Bernstein DI, Riedesel JM, Lan D, Huang B, et al. Pediatricians' intention to administer human papillomavirus vaccine: the role of practice characteristics, knowledge, and attitudes. J Adolesc Health. 2005;37(6):502-10

31. Vadaparampil ST, Malo TL, Sutton SK, Ali KN, Kahn JA, Casler A, et al. Missing the target for routine human papillomavirus vaccination: consistent and strong physician recommendations are lacking for 11- to 12-year-old males. Cancer Epidemiol Biomark Prev. 2016;25(10):1435-46.

32. White A, Hingson R. The burden of alcohol use: excessive alcohol consumption and related consequences among college students. Alcohol Res. 2013;35(2):201-18.

33. Metrik J, Caswell AJ, Magill M, Monti PM, Kahler CW. Sexual risk behavior and heavy drinking among weekly marijuana users. J Stud Alcohol Drugs. 2016;77(1):104-12.

\section{Publisher's Note}

Springer Nature remains neutral with regard to jurisdictional claims in published maps and institutional affiliations.
Ready to submit your research? Choose BMC and benefit from:

- fast, convenient online submission

- thorough peer review by experienced researchers in your field

- rapid publication on acceptance

- support for research data, including large and complex data types

- gold Open Access which fosters wider collaboration and increased citations

- maximum visibility for your research: over $100 \mathrm{M}$ website views per year

At BMC, research is always in progress.

Learn more biomedcentral.com/submissions 